\title{
Sporting Injuries - An Unusual Case of a Traumatic Schwannoma Presenting on the Upper Lip of a Professional Footballer, a Case Report
}

\author{
Mohamed Hania $^{\mathrm{a}, \mathrm{b}}$, Christopher Mannion ${ }^{\mathrm{a}}$
}

\begin{abstract}
Schwannomas are benign tumors that typically arise from the perineural Schwann cells responsible for the production of the insulting sheath called myelin with only $1 \%$ occurring intraorally. We present and report a rare case of a schwannoma presenting on the upper lip of a 29-year-old male professional footballer who was referred to OMFS clinic after with a persistent swelling on his upper lip following trauma to the face 1 year ago. The treatment for benign schwannoma consists of total surgical excision. However, recurrences as well as the malignant transformation are rare events. It is possible that other disorders may appear with similar clinical picture, so the need for a biopsy of the suspected lesion cannot be emphasized enough to rule out other conditions. The differential diagnoses of schwannomas include lesions such as lipomas, hemangiomas, eosinophilic granuloma, epidermoid and dermoid cysts, epithelial hyperplasia, granular cell tumour, leiomyoma, and lymphangioma.
\end{abstract}

Keywords: Schwannoma; Lip swelling; Sporting injuries; Maxillofacial surgery; Excision biopsy; Trauma; Soft tissue swelling

\section{Introduction}

Schwannomas are benign tumors that typically arise from the perineural Schwann cells responsible for the production of the insulting sheath called myelin. They may develop at any age but are most common in younger to middle aged adults but unclear gender predisposition [1]. They are relatively slow growing masses with a strong predilection for sensory nerves. Schwannoma are mostly benign; however, less than $1 \%$ can underdevelop in a malignancy called neurofibrosarcoma.

There are $25-50 \%$ of schwannomas occurring in the head and neck area [2] and only 1\% occurring intraorally. Though the majority of schwannomas are idiopathic, trauma is sug-

Manuscript submitted January 24, 2017, accepted February 6, 2017

aOMFS Leeds General Infirmary, Leeds, UK

${ }^{b}$ Corresponding Author: Mohamed Hania, OMFS Leeds General Infirmary, Leeds, UK. Email: mhania@tcd.ie

doi: https://doi.org/10.14740/jmc2761w gested as a factor in papers written by Wilkinson et al [3] and Brody et al [4] in vertebral fracture and thyroidectomy, respectively. Schwannomas may also be associated with certain syndromes, such as neurofibromatosis type 2 and schwannomatosis in certain cases [5].

Diagnosis is centered on biopsy with microscopic features of solitary, well-circumscribed, encapsulated lesions confined to the subcutis. Characteristic presences include Antoni A and Antoni B areas. Antoni A is composed of spindle-shaped Schwann cells in an interlacing fascicle with Antoni B consisting of loose meshwork of gelatinous and microcytic tissue with large, irregular spaced, thick-walled vessels. Mitotic features may also be present. S100 protein is strongly expressed by most cells of schwannoma along with vimentin and myelin.

MRIs are the first-line imaging of choice as they have fairly predictable signal characteristics: T1 is either isointense or hypointense, T1 C+ (Gd) intense enhancement, T2 heterogeneously hyperintense (Antoni A: low; Antoni B: high), T2*: hemosiderin present in larger tumors, along with useful signs such as split-fat, target and fascicular sign.

We present and report a rare case of a schwannoma presenting on the upper lip.

\section{Case Report}

A 29-year-old male professional footballer presented to our OMFS outpatient clinic after being referred by his GP due to a persistent swelling on his upper lip.

The patient presented with a swelling on the upper lip, which had been present for over 2 years. He associated this with an accidental elbowed to the face in a football match by an opponent. The swelling had been asymptomatic since but in the past 3 months, the patient noticed an increase in size of lesion, which was of concern to the patient. He is fit and well individual with no underlying medical condition and topical antibiotic cream for his acne was noted.

On examination, a $3 \mathrm{~cm}$ blueish-tinge, firm mobile but well-circumscribed swelling was noted on right side of the upper lip midline with a submucosal component as well (Fig. 1). Clinical photography and an incisional biopsy were arranged for the patient.

The incisional biopsy was completed under local anesthetic by linear incision into the labial mucosa and blunt dissection submucosal towards the swelling. The lesion was observed to 


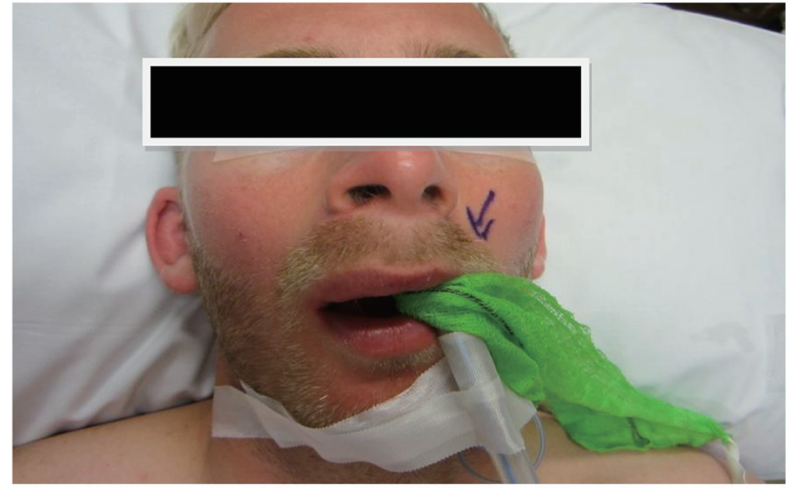

Figure 1. Extra-oral view of lip swelling.

be solid, white in color and non-cystic. A specimen was sent to our pathology laboratory and biopsy site was closed with 4.0 vicryl rapide.

Two weeks later, the pathology result revealed a "wellcircumscribed subcutis with intersected bundles of monomorphic spindle cell with focal palisading", i.e. schwannoma. The patient was informed of the pathology result with a proposed plan of excisional biopsy of the lesion under general anesthetic.

The excisional biopsy was completed via an intraoral approach with access to the lesion achieved by blunt dissection following elliptical mucosal incision along previous biopsy site (Fig. 2). The lesion was delivered whole with overlying mucosal tissue and measured $3 \times 2.5 \times 1.7 \mathrm{~cm}$ (Fig. 3). The surgical site was closed in levels and the patient made an uneventful full recovery. The second biopsy result confirmed the previous diagnosis of schwannoma.

At 6-week follow-up, the patient had experienced mild hyperexcitability of sensory nerves characterized by an "itch" feeling and slight unevenness of the upper lip on smile. However, these issues have been completely resolved at 6 months review.

\section{Discussion}

We hypothesize that a previous impact injury to the upper lip as result of his profession may be the cause of his development

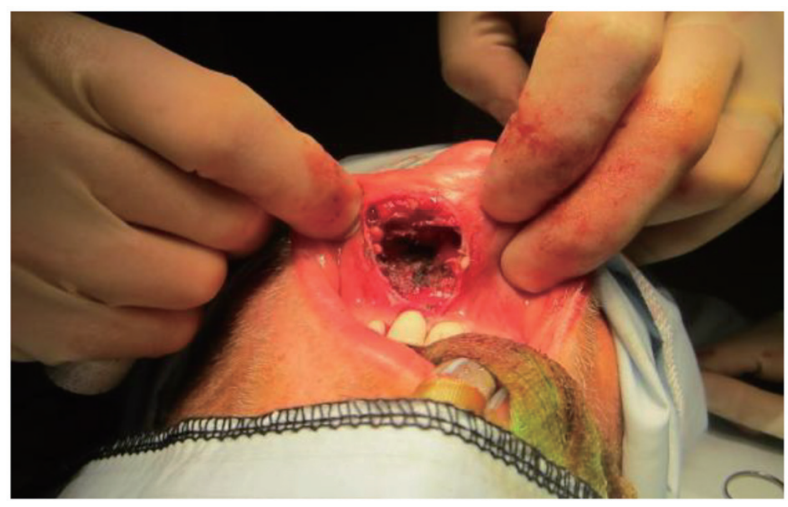

Figure 2. Surgical site after excision of lesion.

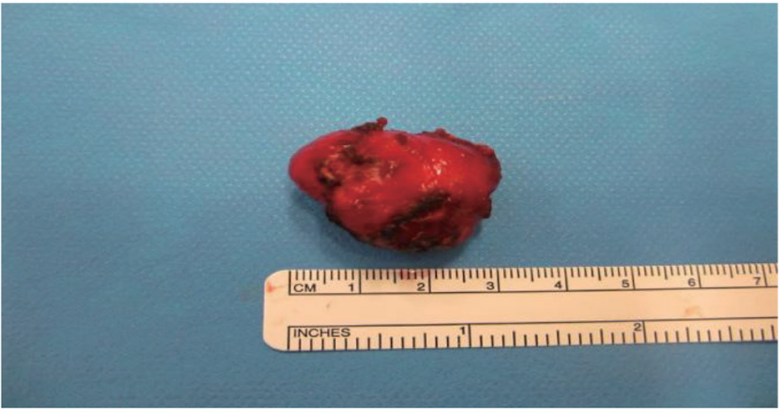

Figure 3. Excised schwannoma.

of this tumor.

This case report represents a rare condition in itself but we also found less than 20 cases of schwannomas occurring on the lip that has been reported in the English literature.

Less than $1 \%$ of schwannomas occur intraorally and they develop $45-52 \%$ on the tongue, $13 \%$ on the cheek, $20 \%$ on buccal/vestibular mucosa and $19 \%$ on lip and gingivae [6].

It is possible that other disorders will appear with similar clinical picture, so the need for a biopsy of the suspected lesion cannot be emphasized enough to rule out other conditions.

The differential diagnoses of schwannomas include lesions such as lipomas, hemangiomas, eosinophilic granuloma, epidermoid and dermoid cysts, epithelial hyperplasia, granular cell tumor, leiomyoma, and lymphangioma.

The treatment for benign schwannoma consists of total surgical excision. Ultrasonography, CT, and MRI may be helpful diagnostic and treatment tools, for the estimation of tumor margins, the lesion composition, and the determination of whether there is infiltration to surrounding structures [7]. The recurrences as well as the malignant transformation are rare events.

However, a differentiation between schwannoma and neurofibroma is important, as the latter may be a manifest of neurofibromatosis which may precipitate a more thorough examination of the other parts of the body [8].

A better understanding of trauma-induced schwannoma, especially intraorally, may add to the clinical care of patients with this rare disorder as this is a lesion not encountered often in practice.

\section{References}

1. Enzinger IM, Weiss SW. Soft tissue tumour, 3rd ed. St Louis: MO Mosby; 1995. p. 821-850.

2. Ducatman BS, Scheithauer BW, Piepgras DG, Reiman HM, Ilstrup DM. Malignant peripheral nerve sheath tumors. A clinicopathologic study of 120 cases. Cancer. 1986;57(10):2006-2021.

3. Wilkinson JM, McClelland MR, Battersby RD. Spinal cord schwannoma after vertebral trauma: a causal relation? J Neurol Neurosurg Psychiatry. 1995;59(4):358.

4. Kennedy WP, Brody RM, LiVolsi VA, Wang AR, Mirza NA. Trauma-induced schwannoma of the recurrent laryngeal nerve after thyroidectomy. Laryngoscope. 2016;126(6):1408-1410. 
5. Hilton DA, Hanemann CO. Schwannomas and their pathogenesis. Brain Pathol. 2014;24(3):205-220.

6. Baderca F, Cojocaru S, Lazar E, Lazureanu C, Faur A, Lighezan R, Alexa A, et al. Schwannoma of the lip: case report and review of the literature. Rom J Morphol Embryol. 2008;49(3):391-398.
7. Asaumi J, Konouchi H, Kishi K. Schwannoma of the upper lip: ultrasound, CT, and MRI findings. J Oral Maxillofac Surg. 2000;58(10):1173-1175.

8. Williams HK, Cannell H, Silvester K, Williams DM. Neurilemmoma of the head and neck. Br J Oral Maxillofac Surg. 1993;31(1):32-35. 